\title{
Correction calculation of the heat source power during plasma-powder surfacing of the necks of stepped shafts
}

\author{
G.R. Latypova ${ }^{1}$, N.N. Karpenko ${ }^{1}$, and R.A. Latypov ${ }^{1, *}$ \\ ${ }^{1}$ Moscow Polytechnic University, street Bolshaya Semenovskaya street, 38, Moscow, Russia
}

\begin{abstract}
A mathematical model is proposed for calculating temperature fields during arc surfacing of limited cylinders, eliminating the need for dimensionless parameters, when solving the differential heat equation, which facilitates the practical application of the developed calculation method. It is proposed that the penetration depth be calculated using the Lambert function. The obtained dependences make it possible to quantify the required correction of the heat source power in the process of surfacing the necks of stepped shafts.
\end{abstract}

\begin{abstract}
Plasma-powder surfacing is one of the most common methods of hardening and restoring the working surfaces of the "shaft" type parts []. In the process of surfacing, a general heating of the part occurs, which leads to a change in the geometric parameters of the weld pool, in particular, to an increase in its width and depth of penetration. The consequence of this is a change in the chemical composition of the weld metal due to an increase in the proportion of the base metal in the weld pool, a change in cooling rates and, accordingly, the resulting structure of the weld metal. It follows that to ensure the specified quality of the workpieces, it is necessary to maintain the stability of the geometric parameters of the weld pool during surfacing. This task is especially relevant when surfacing the necks of stepped and limited shafts, in which the surface reflection of heat flows has a significant effect on the distribution of temperature fields. One of the ways to stabilize the size of the weld pool is to correct the power of the heat source depending on the average temperature of the part. In order to reduce the cost of conducting preliminary experiments in the development of technologies for surfacing specific parts, it seems appropriate to carry out preliminary mathematical modeling of the thermal situation in the field of formation of the weld metal roller. Most fully, this problem can be solved by numerical methods for solving the heat problem, for example, by the finite element method. However, this is associated with a significant expenditure of machine time. In this regard, for the initial assessment of the thermal situation, it seems advisable to develop simpler methods for solving the thermal problem based on well-known analytical solutions of the differential heat equation. In particular, mathematical models are known that schematize the deposited part in the form of an endless cylinder. The modeling of temperature fields as applied to the arc surfacing of unilaterally bounded cylinders was considered in works $[1,4,5]$. The solutions of this
\end{abstract}

* Corresponding author: latipov46@mail.ru 
particular case, obtained in these works, use dimensionless parameters, which complicates their practical application in the development of shaft arc surfacing technologies. The case of modeling temperature fields during surfacing of bilaterally limited cylinders remains little studied.

The calculation scheme for modeling temperature fields during surfacing of bilaterally limited cylinders is shown in Fig. 1.

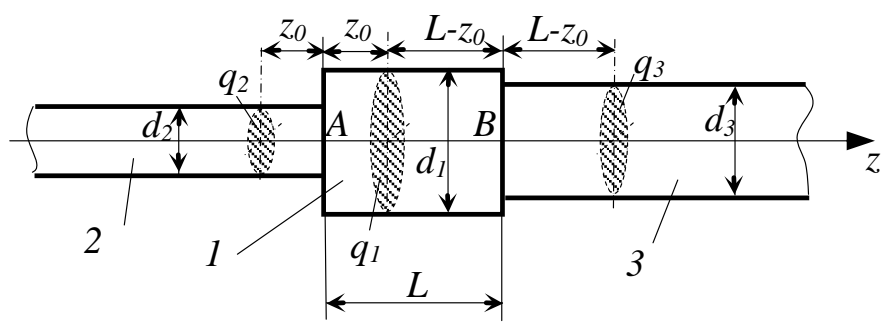

Fig. 1. Calculation scheme for modeling temperature fields during surfacing of a stepped shaft neck

A neck 1 of length $\quad L$ and diameter $d_{1}$ is surfaced. Neck 1 is paired with neck 2 and 3 by diameters $d_{2}$ and $d_{3}$ respectively, at the same time $d_{1} \geq d_{2}$ and $d_{1} \geq d_{3}$. A surface point source with power $q_{1}$ moves along a helix from point $A$ to point $B$.

Following the works $[1,4]$, the temperature field of a circular cylinder, fused along a helical line, is represented as the sum of two temperature fields:

$$
T=T_{s}+T_{q}
$$

where $T$ - temperature at an arbitrary point in a circular cylinder, $T_{s}$ - temperature due to heating of the part from previous turns, $T_{q}$ - temperature field around a point heat source.

When calculating the temperature $T_{s}$ the surface heat source is replaced by a flat heat source. From simple estimates of the heat transfer process, we obtained that the adoption of this assumption will be more justified, the more fully the requirement is met:

$$
\frac{m V}{4 \pi^{2} a}<1
$$

where $m, V$ - step and speed of surfacing, $a$ - thermal diffusivity coefficient.

To take into account the influence of the neck 1 ends on the features of its heating, we apply the principle of a fictitious heat source [6]. So, the temperature of an arbitrary section of the neck 1 , remote from the end face $A$ by a distance $z_{0}$, will be determined by the action of the heat source $q_{1}$, source $q_{2}$, located symmetrically to the end face $A$, and source $q_{3}$, located symmetrically to the end face $B$ (fig. 1 ). We estimate the power of fictitious sources $q_{2}$ and $q_{3}$ as follows. Obviously, if the cross-sectional areas of adjacent necks

$$
\frac{\pi d_{2}^{2}}{4}=\frac{\pi d_{3}^{2}}{4}=0
$$

then

$$
q_{2}=q_{3}=q_{1}
$$


If

$$
\frac{\pi d_{2}^{2}}{4}=\frac{\pi d_{3}^{2}}{4}=\frac{\pi d_{1}^{2}}{4},
$$

then

$$
q_{2}=q_{3}=0
$$

The power change $q_{2}$ and $q_{3}$ in the ranges $d_{2}$ and $d_{3}$ from 0 to $d_{1}$ is interpolated by linear dependencies. Based on dependencies (3-6), the following formulas were obtained:

$$
\begin{aligned}
& q_{3}=q_{1}\left(1-\frac{d_{3}^{2}}{d_{1}^{2}}\right), \\
& q_{2}=q_{1}\left(1-\frac{d_{2}^{2}}{d_{1}^{2}}\right) .
\end{aligned}
$$

As a result, taking into account (7) and (8), we obtained:

$$
\begin{aligned}
& T_{S}(z, t)=\int_{0}^{t} \frac{2 q_{1}}{\pi c \rho d_{1}^{2} \sqrt{\pi a\left(t-t^{\prime}\right)}} \exp \left[-\frac{\left(z_{0}-v t^{\prime}\right)^{2}}{4 a\left(t-t^{\prime}\right)}\right] d t^{\prime}+ \\
& +\int_{0}^{t} \frac{2 q_{1}\left(1-\frac{d_{3}^{2}}{d_{1}^{2}}\right)}{\pi c \rho d_{1}^{2} \sqrt{\pi a\left(t-t^{\prime}\right)}} \exp \left[-\frac{\left(2 L-z_{0}-v t^{\prime}\right)^{2}}{4 a\left(t-t^{\prime}\right)}\right] d t^{\prime}+, \\
& +\int_{0}^{t} \frac{2 q_{1}\left(1-\frac{d_{2}^{2}}{d_{1}^{2}}\right)}{\pi c \rho d_{1}^{2} \sqrt{\pi a\left(t-t^{\prime}\right)}} \exp \left[-\frac{\left(z_{0}+v t^{\prime}\right)^{2}}{4 a\left(t-t^{\prime}\right)}\right] d t^{\prime}
\end{aligned}
$$

where $c \rho$ - specific heat, $\quad v$-speed of a flat source movement, $t$ - time.

The speed of a flat source movement is

$$
v=\frac{m V}{\pi d_{1}} .
$$

Integration time is

$$
t=\frac{z_{0}}{v}
$$

The power of a flat heat source $q_{1}$ was calculated by the formula

$$
q_{1}=\eta I U
$$

where $\eta$ - arc efficiency, $I$ - arc current, $U$ - arc voltage.

We will find the temperature $T_{q}$ by applying the action scheme of a point source on a semi-infinite body while neglecting the curvature of the part's surface compared to the size 
of the weld pool. In a moving coordinate system, the temperature field of the limiting state when a surface of a semi-infinite body is heated by a point source is [6]

$$
T_{q}(r, t)=T_{0}+\frac{q}{2 \pi \lambda R} \exp \left[-\frac{V}{2 a}(r+x)\right]
$$

where $r=\sqrt{x^{2}+y^{2}+z^{2}}$ - length of the radius vector of the considered point of a semiinfinite body in a moving coordinate system; $T_{0}$ - initial temperature, in this case $T_{0}=T_{S}(z, t)$.

The power of the heat source $q$ was found taking into account the consumption of thermal energy of the plasma arc on the heated powder

$$
q=\eta I U-p_{p} c_{p}\left(T_{p, f}-T_{p, 0}\right),
$$

where $p_{p}, c_{p}, T_{p, f}, T_{p, 0}$ - flow rate, heat capacity, melting point and initial temperature of the weldable powder material, respectively.

We find the temperature distribution in normal $y$ to the surface at the point of the heat source action, assuming $x=z=0$. Then expression (1) will take the form:

$$
T(R, t)=T_{S}(z, t)+\frac{q}{2 \pi \lambda y} \exp \left[-\frac{V y}{2 a}\right],
$$

where $\lambda$ - thermal diffusivity coefficient.

We find the penetration depth $y_{f}$ from (15), believing $T(r, t)=T_{f}$

$$
y_{f}=\frac{2 a}{V} \text { LambertW }\left(\frac{V q}{4 \pi a \lambda\left(T_{f}-T_{S}\right)}\right) \text {, }
$$

where $T_{f}$ - melting temperature, LambertW $(X)$ - Lambert function.

For an approximate calculation of the Lambert function, we use the well-known dependence [7]

$$
\text { LambertW }(X)=0,665[1+0,0195 \ln (X+1)] \ln (X+1)+0,04 \text {. }
$$

Then

$$
y_{f}=\frac{0,6652 a}{V}\left[1+0,0195 \ln \left(\frac{V q}{4 \pi a \lambda\left(T_{f}-T_{S}\right)}+1\right)\right] \ln \left(\frac{V q}{4 \pi a \lambda\left(T_{f}-T_{S}\right)}+1\right)+0,04
$$

From (15) it follows that in order to maintain a constant penetration depth $y_{f}$, the current power of the heat source must be

$$
q=\frac{2 \pi \lambda y\left(T_{f}-T_{s}\right)}{\exp \left[-\frac{V y_{f}}{2 a}\right]} .
$$


Dependence (19) can be used to calculate the required correction of the heat source power in the process of surfacing the stepped shafts necks.

\section{References}

1. Nefedov B.B. Thermal efficiency of plasma-powder surfacing during restoration of agricultural machinery parts: dissertation ... Doctor of technical sciences: 05.20.03. Moscow, 2002. - 380 p.

2. Y. H. Lv et al., Plasma Transferred Arc Powder Surfacing Technology of Thrust Face, Key Engineering Materials, Vols. 373-374, pp. 43-46, 2008

3. A. Klimpel, L. A. Dobrzański, A. Lisiecki, and D. Janicki, The study of the technology of laser and plasma surfacing of engine valves face made of X40CrSiMo10-2 steel using cobalt-based powders, J. Mater. Proc. Tech., 175, Issues 1-3, 251-256 (2006).

4. Makhnenko V.I., Kravtsov T.G. Thermal processes during mechanized surfacing of parts such as circular cylinders. Kiev: Naukova Dumka, 1976.- 160 p.

5. Yazovskikh V.M., Belenky V.Ya. Thermal processes during surfacing of continuous cylindrical bodies // Welding and diagnostics. 2011. No 3. Pp. 27-31.

6. Theory of welding processes. M.: Publishing House of MSTU. N.E. Bauman, 2007 -752 p.

7. Dubinov A.E., Dubinov I.D., Saykov S.K. Lambert W-function and its application in mathematical problems of physics: Textbook. manual for universities. Sarov: FSUE RFNC-VNIIEF, 2006.- 160 p. 